PSFC/JA-10-5

\title{
Fluid and Drift-Kinetic Description of a Magnetized Plasma with \\ Low Collisionality and Slow Dynamics \\ Orderings. I: Electron Theory
}

J.J. Ramos

March, 2010

\author{
Plasma Science and Fusion Center \\ Massachusetts Institute of Technology \\ Cambridge MA 02139, U.S.A.
}

This work was supported by the U.S. Department of Energy under Grants Nos. DEFG02-91ER54109, DEFC02-08ER54969 and DEFC02-06ER54855. Reproduction, translation, publication use and disposal, in whole or in part, by or for the United States government is permitted. 


\title{
Fluid and Drift-Kinetic Description of a Magnetized Plasma with Low Collisionality and Slow Dynamics Orderings. I: Electron Theory.
}

\author{
J.J. Ramos
}

Plasma Science and Fusion Center, Massachusetts Institute of Technology, Cambridge MA, U.S.A.

\begin{abstract}
A closed theoretical model to describe slow, macroscopic plasma processes in a fusion-relevant collisionality regime is put forward. This formulation is a hybrid one, with fluid conservation equations for particle number, momentum and energy, and drift-kinetic closures. Intended for realistic application to the core of a high-temperature tokamak plasma, the proposed approach is unconventional in that the ion collisionality is ordered lower than in the ion banana regime of neoclassical theory. The present first part of a two-article series concerns the electron system, which is still equivalent to one based on neoclassical electron banana orderings. This system is derived such that it ensures the precise compatibility among the complementary fluid and drift-kinetic equations, and the rigorous treatment of the electric field and the Fokker-Planck-Landau collision operators. As an illustrative application, the special limit of an axisymmetric equilibrium is worked out in detail.
\end{abstract}




\section{Introduction.}

Macroscopic processes in a magnetized plasma, defined as those whose characteristic length scales are always greater than the ion Larmor gyroradius, are amenable to a fluid description of their dynamics perpendicular to the magnetic field, closed by a drift-kinetic solution for their parallel dynamics ${ }^{1,2}$. This formulation is based on asymptotic expansions of the underlying Vlasov-Boltzmann kinetic equations and their velocity moments in powers of the small ratio $\delta \sim \rho_{\iota} / L_{\perp} \ll 1$ between the ion gyroradius and the smallest macroscopic length scale, typically established by a perpendicular gradient or fluctuation wavenumber. Consideration of the gyroradius parameter $\delta$ alone is sufficient to carry out this theory in the case of a strictly collisionless plasma. However, the occurrence of collisions introduces a second fundamental parameter, namely the ratio $\nu_{*} \sim L_{\|} / \lambda^{\text {coll }}$ between the characteristic macroscopic parallel length and the characteristic collisional length $\lambda^{\text {coll }} \sim v_{t h s} / \nu_{s}$, with $v_{t h s}$ and $\nu_{s}$ respectively the thermal velocity and collision frequency of species $s$ assumed all to have comparable temperatures. In the core plasma of a typical tokamak magnetic fusion experiment, the parameter $\nu_{*}$ cannot be neglected altogether but it is much smaller than unity. Some important macroscopic phenomena in these magnetic fusion plasmas, such as the precursor of the sawtooth internal disruption ${ }^{3,4}$ and the "neoclassical tearing" mode ${ }^{5,6}$, develop on time scales of the order of the diamagnetic drift scale or slower, i.e. frequencies of order $\delta$ or smaller relative to the Alfven or sound frequencies characteristic of single-fluid magnetohydrodynamics (MHD). The analysis of these slow modes in a hot magnetic fusion plasma, which is the main motivation for the present work, requires therefore a careful consideration of the relative orderings between the two small parameters $\delta$ and $\nu_{*}$. In addition, the specific ordering of the small mass ratio between electrons and ions (which is not essential in a strictly collisionless theory) becomes essential when collisions are taken into account.

Given the formal freedom to order the parameters $\delta, \nu_{*}$ and $m_{e} / m_{\iota}$ (plus several other plausible expansion parameter choices such as geometrical ratios or the pressure ratio $\beta$ ), many different models have been constructed as exemplified by Refs.6-17. However, apart from the strictly collisionless formulations, all mainstream finite collisionality models share, to this author's best knowledge, one common feature: the collisionality parameter $\nu_{*}$ is always ordered as comparable or higher than in 
the lowest ion collisionality regime of neoclassical theory and this turns out to be unrealistically high for any fusion-grade tokamak plasma. Low collisionality (so called banana regime) ion neoclassical theory ${ }^{18-20}$ assumes both $\delta$ and $\nu_{*}$ to be much less than unity and carries out a primary drift-kinetic expansion to first order in $\delta$, followed by a subsidiary expansion in $\nu_{*}$, without going to the second order in $\delta$. Thus, even in the least restrictive case where the $\nu_{*}$ expansion is limited to its first order, the validity of this scheme requires $\nu_{*} \gg \delta$ consistent with retaining $O\left(\delta \nu_{*}\right)$ while neglecting $O\left(\delta^{2}\right)$. The condition $\nu_{*} \gg \delta$, inherent to any low collisionality theory that, like the neoclassical one, does not consider a second order ion drift-kinetic equation, is easily violated by any modern tokamak core plasma with ion temperatures in the several $\mathrm{KeV}$ range. Instead, as proposed in a previous study of the fluid moment equations ${ }^{21}$, the present work will assume the alternative, lower collisionality ordering $\nu_{*} \sim \delta$. This implies forsaking a hypothetical unified connection with the intermediate and high collisionality regimes $\nu_{*} \gtrsim 1$ (which, barring a solution of the complete primary kinetic equations, appears impossible to achieve consistently anyway) in favor of a more realistic description of the high temperature regime of interest. In addition, the rather small electron to ion mass ratio is to be formally ordered as comparable to $\delta^{2}$. A systematic theory can then be built based on the somewhat unconventional but arguably fusion-relevant asymptotic expansion in uniform powers of $\delta \sim \nu_{*} \sim\left(m_{e} / m_{\iota}\right)^{1 / 2} \ll 1$. In the interest of obtaining clear-cut results from this basic single-parameter ordering scheme, no further assumptions involving additional expansion parameters will be made. In particular, all macroscopic length scales will be formally taken as comparable, i.e. $L_{\perp} \sim L_{\|} \sim L$, and the ratio $\beta$ between thermal and magnetic pressures will be formally taken as order unity. These maximal orderings could be relaxed a posteriori with subsidiary expansions in $L_{\perp} / L_{\|} \ll 1$ or $\beta \ll 1$ depending on specific applications. In accordance with its intended goal of describing slow excursions from magnetically confined equilibria, the theory to be developed here will assume close to Maxwellian distribution functions ${ }^{2}$. Then, the orderings of the non-Maxwellian perturbations in terms of the basic expansion parameter $\delta$ will be dictated by compatibility with a consistent perturbative solution of the kinetic equations.

It is planned to present this work in a set of two articles, the first one devoted to the electrons and the second one to the ions. According to the proposed ordering scheme, the electron gyroradius parameter is $\delta_{e} \sim \rho_{e} / L \sim\left(m_{e} / m_{\iota}\right)^{1 / 2} \delta \sim \delta^{2}$. The reach of the analysis will extend to the inverse time 
scale of order $\delta^{3} v_{\text {the }} / L$, where the collisional dynamics begins to play a role. Thus, effects of order $\delta_{e} \nu_{*} \sim \delta^{3}$ will be included whereas effects of order $\delta_{e}^{2} \sim \delta^{4}$ will be neglected and the electron formulation will be equivalent to one based on neoclassical electron banana orderings. On the other hand, when expressed in terms of the ion thermal velocity, the collisional inverse time scale of interest is $O\left(\delta^{3} v_{t h e} / L\right)=O\left(\delta^{2} v_{t h \iota} / L\right)$. This means that effects of order $\delta \nu_{*} \sim \delta^{2}$ are to be included for the ions, which will necessitate a second order ion drift-kinetic equation and a departure from conventional neoclassical theory. In another departure from the more traditional approaches, the electron and ion distribution functions and their drift-kinetic equations will be expressed in the moving reference frames of the corresponding species macroscopic flow. This facilitates the evaluation of the moments needed to close the fluid equations and ensures the precise compatibility between the complementary fluid and drift-kinetic systems as well as the exact treatment of the electric field. Finally, due to the assumed low collisionality and near Maxwellian orderings, only the linearized version of the collision operators will be needed through the $O\left(\delta^{3} v_{\text {the }} / L\right)$ scale to be considered. However, the complete Fokker-Planck-Landau ${ }^{22}$ forms for Coulomb collisions will be retained and care will be exercised when carrying out the small mass ratio reductions, in order to avoid spurious results that sometimes arise as the consequence of oversimplified collision operator models.

\section{General framework and asymptotic ordering scheme.}

The theory to be developed here assumes a quasineutral plasma, made for simplicity of electrons and a single ion species of unit charge, with comparable temperatures. It also assumes that the electron and ion physics is described by one-particle distribution functions that evolve according to Vlasov-Boltzmann kinetic equations with Coulomb collision operators in their Fokker-Planck-Landau form. This kinetic system will be expanded asymptotically in powers of the small ion gyroradius fundamental parameter:

$$
\delta \sim \rho_{\iota} / L \ll 1
$$

with small mass ratio and low collisionality orderings linked to $\delta$ :

$$
\left(m_{e} / m_{\iota}\right)^{1 / 2} \sim \delta, \quad \text { hence } \quad \delta_{e} \sim \rho_{e} / L \sim \delta^{2}
$$


and

$$
\nu_{\iota} \sim \delta \nu_{e} \sim \delta v_{t h \iota} / L \sim \delta^{2} v_{\text {the }} / L \sim \delta^{2} \Omega_{c \iota}, \quad \text { hence } \quad \lambda^{\text {coll }} \sim v_{t h \iota} / \nu_{\iota} \sim v_{\text {the }} / \nu_{e} \sim \delta^{-1} L
$$

Here, $\nu_{s}, \Omega_{c s}$ and $v_{t h s}$ stand, respectively, for the collision frequency, cyclotron frequency and thermal velocity of species $s$. The macroscopic flows will be assumed to be of the order of the diamagnetic drifts, i.e. $u_{\iota} \sim u_{e} \sim u_{D \iota, e} \sim \delta v_{t h \iota} \sim \delta^{2} v_{t h e}$. As previously discussed, no ordering asumptions will be made regarding macroscopic length ratios or the pressure ratio $\beta$.

The processes this theory is intended to apply to are slow excursions from a magnetically confined equilibrium, hence the distribution functions, $f_{s}$, can be assumed to be close to Maxwellians ${ }^{2}$ :

$$
f_{s}=f_{M s}+f_{N M s}=\frac{n}{(2 \pi)^{3 / 2} v_{t h s}^{3}} \exp \left(-\frac{\left|\mathbf{v}-\mathbf{u}_{s}\right|^{2}}{2 v_{t h s}^{2}}\right)+f_{N M s},
$$

with Maxwellian parts, $f_{M s}$, that are referred to the moving frames of the corresponding macroscopic flows, $\mathbf{u}_{s}$, and small non-Maxwellian perturbations, $\left|f_{N M s}\right| \ll\left|f_{M s}\right|$. The thermal velocities are defined as $v_{t h s}^{2} \equiv T_{s} / m_{s} \equiv p_{s} /\left(m_{s} n\right)$, where $T_{s}$ and $p_{s}$ are the mean temperature and pressure of species $s$, and $n$ is the particle number density. This near-Maxwellian ansatz is consistent with an asymptotic solution of the kinetic equations under the above low collisionality orderings, provided the temperature gradients parallel to the magnetic field $\mathbf{B}$ are small

$$
\mathbf{B} \cdot \nabla T_{s} \sim \delta^{2} B T_{s} / L
$$

with the non-Maxwellian perturbations ordered as

$$
f_{N M \iota} \sim \delta f_{M \iota} \quad \text { and } \quad f_{N M e} \sim \delta^{2} f_{M e}
$$

Like the Maxwellian parts, the non-Maxwellian parts of the distribution functions will be evaluated in the moving reference frames of the macroscopic flows. Then, the $1, \mathbf{v}-\mathbf{u}_{s}$ and $\left|\mathbf{v}-\mathbf{u}_{s}\right|^{2}$ velocity moments of $f_{N M s}$ will be required to vanish and, in Chapman-Enskog-like fashion ${ }^{23}$, the density, flow velocities and temperatures will be carried entirely by the Maxwellians. The hybrid fluid and drift-kinetic dynamical system will consist of the fluid moment equations for the density, flow velocities and temperatures, complemented by drift-kinetic equations for $f_{N M s}$ whose solutions provide the variables needed to close the fluid equations. A clear advantage of obtaining these non-Maxwellian 
solutions in the reference frames of the macroscopic flows is that the evaluation of the closure variables such as the stress and heat flux tensors is then direct, without the need of substracting the mean flows.

With the adopted low collisionality and close to Maxwellian orderings, the electron collision operators are $C_{e e}\left[f_{e}, f_{e}\right] \sim C_{e \iota}\left[f_{e}, f_{\iota}\right] \sim \delta^{2} \nu_{e} f_{M e} \sim \delta^{3}\left(v_{t h e} / L\right) f_{M e}$. Similarly, the ion collision operators are $C_{\iota \iota}\left[f_{\iota}, f_{\iota}\right] \sim C_{\iota e}\left[f_{\iota}, f_{e}\right] \sim \delta \nu_{\iota} f_{M \iota} \sim \delta^{2}\left(v_{t h \iota} / L\right) f_{M \iota} \sim \delta^{3}\left(v_{t h e} / L\right) f_{M \iota}$. Therefore, the collisional effects will begin to influence the dynamics on the inverse time scale of order $\delta^{3} v_{t h e} / L \sim \delta^{3} \Omega_{c \iota}$, which is the smallest frequency scale the analysis will be carried to. Before this third order collisional scale is reached, the second order relative to the ion cyclotron frequency corresponds to the diamagnetic drift frequency scale, $\omega_{D \iota, e}=u_{D \iota, e} / L \sim u_{\iota, e} / L \sim \delta^{2} \Omega_{c \iota}$, and the first order corresponds to the MHD frequency scale (Alfven and sound for $\beta \sim 1$ ), $\omega_{A}=c_{A} / L \sim \omega_{S}=c_{S} / L \sim v_{t h \iota} / L \sim \delta \Omega_{c \iota}$.

\section{Electron fluid system.}

The electron fluid system will consist of the quasineutral Maxwell equations for the electromagnetic fields $(\mathbf{E}, \mathbf{B})$ plus the three moments of the electron kinetic equation that evolve the macroscopic density, flow velocity and temperature. Thus, to all asymptotic orders considered here,

$$
\begin{gathered}
\frac{\partial \mathbf{B}}{\partial t}=-\nabla \times \mathbf{E}, \\
\mathbf{j}=\operatorname{en}\left(\mathbf{u}_{\iota}-\mathbf{u}_{e}\right)=\nabla \times \mathbf{B}
\end{gathered}
$$

and

$$
\frac{\partial n}{\partial t}+\nabla \cdot\left(n \mathbf{u}_{e}\right)=0
$$

The remaining two (momentum conservation and temperature) fluid equations will be taken in their

third order approximation under the $\delta \sim \nu_{*} \sim\left(m_{e} / m_{\iota}\right)^{1 / 2} \sim\left|\mathbf{u}_{e} / v_{\text {the }}\right|^{1 / 2} \sim\left|f_{N M e} / f_{M e}\right|^{1 / 2}$ asymptotic expansion this work is based on. Expanding the $m_{e} \mathbf{v}$ moment of the electron Vlasov-Boltzmann equation (see. e.g. Ref.21) and retaining terms to $O\left(\delta^{3} n m_{e} v_{\text {the }}^{2} / L\right)$ while neglecting $O\left(\delta^{4} n m_{e} v_{\text {the }}^{2} / L\right)$, one gets

$$
m_{e} n \frac{\partial \mathbf{u}_{e}}{\partial t}+e n\left(\mathbf{E}+\mathbf{u}_{e} \times \mathbf{B}\right)+\nabla\left(n T_{e}\right)+\nabla \cdot\left[\left(p_{e \|}-p_{e \perp}\right)(\mathbf{b} \mathbf{b}-\mathbf{I} / 3)\right]-\mathbf{F}_{e}^{\text {coll }}=0
$$


where $p_{e \|}$ and $p_{e \perp}$ are the parallel and perpendicular pressures, $n T_{e}=p_{e}=\left(p_{e \|}+2 p_{e \perp}\right) / 3$ is the mean pressure, $\mathbf{F}_{e}^{\text {coll }}$ is the collisional friction force, $\mathbf{b}=\mathbf{B} / B$ is the magnetic unit vector and $\mathbf{I}$ is the identity tensor. Any terms not included in Eq.(10), such as the convective velocity derivative or the divergence of the non-gyrotropic stress tensor, are fourth order or smaller. After a similar expansion of the temperature moment equation, retaining terms to $O\left(\delta^{3} n m_{e} v_{\text {the }}^{3} / L\right)$ while neglecting $O\left(\delta^{4} n m_{e} v_{\text {the }}^{3} / L\right)$, one gets

$$
\frac{3 n}{2} \frac{\partial T_{e}}{\partial t}+\frac{3 n}{2} \mathbf{u}_{e} \cdot \nabla T_{e}+n T_{e} \nabla \cdot \mathbf{u}_{e}+\nabla \cdot\left(q_{e \|} \mathbf{b}-\frac{5 n T_{e}}{2 e B} \mathbf{b} \times \nabla T_{e}\right)-G_{e}^{\text {coll }}=0
$$

where $q_{e \|}$ is the parallel heat flux and $G_{e}^{c o l l}$ is the collisional heat source. Again, any terms not included in Eq.(11), such as the divergence of the collisional perpendicular heat flux or the scalar product of the traceless stress and velocity gradient tensors, are fourth order or smaller. The system (7-11) conserves energy within the retained accuracy of $O\left(\delta^{3} n m_{e} v_{\text {the }}^{2}\right)$ but gives rise to a non-conserved energy residual of $O\left(\delta^{4} n m_{e} v_{t h e}^{2}\right)$. If desired, an exact energy conservation law can be ensured by adding the fourth order terms $m_{e} n\left(\mathbf{u}_{e} \cdot \nabla\right) \mathbf{u}_{e}$ and $\left(p_{e \|}-p_{e \perp}\right)\left\{\mathbf{b} \cdot\left[(\mathbf{b} \cdot \nabla) \mathbf{u}_{e}\right]-\nabla \cdot \mathbf{u}_{e} / 3\right\}$ respectively to Eqs.(10) and (11).

The still unspecified variables needed to close the electron fluid system are the pressure anisotropy $\left(p_{e \|}-p_{e \perp}\right)=\frac{m_{e}}{2} \int d^{3} \mathbf{v}\left\{3\left[\mathbf{b} \cdot\left(\mathbf{v}-\mathbf{u}_{e}\right)\right]^{2}-\left|\mathbf{v}-\mathbf{u}_{e}\right|^{2}\right\} f_{N M e}=O\left(\delta^{2} n m_{e} v_{\text {the }}^{2}\right)+O\left(\delta^{3} n m_{e} v_{\text {the }}^{2}\right)$

the parallel heat flux

$$
q_{e \|}=\frac{m_{e}}{2} \int d^{3} \mathbf{v}\left[\mathbf{b} \cdot\left(\mathbf{v}-\mathbf{u}_{e}\right)\right]\left|\mathbf{v}-\mathbf{u}_{e}\right|^{2} f_{N M e}=O\left(\delta^{2} n m_{e} v_{\text {the }}^{3}\right)+O\left(\delta^{3} n m_{e} v_{t h e}^{3}\right),
$$

and the collisional moments

$$
\mathbf{F}_{e}^{\text {coll }}=m_{e} \int d^{3} \mathbf{v}\left(\mathbf{v}-\mathbf{u}_{e}\right) C_{e \iota}\left[f_{e}, f_{\iota}\right]=O\left(\delta^{3} n m_{e} v_{\text {the }}^{2} / L\right)
$$

and

$$
G_{e}^{\text {coll }}=\frac{m_{e}}{2} \int d^{3} \mathbf{v}\left|\mathbf{v}-\mathbf{u}_{e}\right|^{2} C_{e \iota}\left[f_{e}, f_{\iota}\right]=O\left(\delta^{3} n m_{e} v_{t h e}^{3} / L\right)
$$

These are to be extracted from a drift-kinetic solution for the electron distribution function, which will be the subject of the next two Sections. In addition, the complete closure of Eqs.(7-11) requires knowledge of the macroscopic ion velocity $\mathbf{u}_{\iota}$, that couples this electron system to the analogous ion 
system. For the present purpose of developing the electron side of the theory, the ion velocity (as well as the ion temperature to be needed later) can be considered as given.

\section{Electron drift-kinetic equation.}

Adapting the method developed in Ref.24 for a drift-kinetic equation with fast flow to the problem under consideration here, the electron distribution function will be represented in terms of velocity space coordinates $\left(v_{\|}^{\prime}, v_{\perp}^{\prime}, \alpha\right)$ in the reference frame of the macroscopic flow:

$$
\mathbf{v}=\mathbf{u}_{e}(\mathbf{x}, t)+v_{\|}^{\prime} \mathbf{b}(\mathbf{x}, t)+v_{\perp}^{\prime}\left[\cos \alpha \mathbf{e}_{1}(\mathbf{x}, t)+\sin \alpha \mathbf{e}_{2}(\mathbf{x}, t)\right]
$$

Then, separating its gyrophase-averaged and gyrophase-dependent parts, the distribution function can be written as

$$
f_{e}\left(v_{\|}^{\prime}, v_{\perp}^{\prime}, \alpha, \mathbf{x}, t\right)=\bar{f}_{e}\left(v_{\|}^{\prime}, v_{\perp}^{\prime}, \mathbf{x}, t\right)+\tilde{f}_{N M e}\left(v_{\|}^{\prime}, v_{\perp}^{\prime}, \alpha, \mathbf{x}, t\right)
$$

with

$$
\bar{f}_{e}\left(v_{\|}^{\prime}, v_{\perp}^{\prime}, \mathbf{x}, t\right)=f_{M e}\left(v^{\prime}, \mathbf{x}, t\right)+\bar{f}_{N M e}\left(v_{\|}^{\prime}, v_{\perp}^{\prime}, \mathbf{x}, t\right)
$$

$v^{\prime}=\left(v_{\|}^{\prime 2}+v_{\perp}^{\prime 2}\right)^{1 / 2}$ and $\left\langle\tilde{f}_{N M e}\right\rangle_{\alpha} \equiv(2 \pi)^{-1} \oint d \alpha \tilde{f}_{N M e}=0$.

With the proposed ordering scheme, the non-Maxwellian part of the distribution function is to be solved to the accuracy of $f_{N M e}=O\left(\delta^{2} f_{M e}\right)+O\left(\delta^{3} f_{M e}\right)=O\left(\delta_{e} f_{M e}\right)+O\left(\delta_{e} \nu_{*} f_{M e}\right)$, hence calculations of order $\delta^{4} f_{M e} \sim \delta_{e}^{2} f_{M e}$ are not necessary and a standard drift-kinetic analysis to the first order in $\delta_{e}$ is sufficient. Within this accuracy, the drift-kinetic equation for the gyrophase-averaged part of the distribution function is

$$
\frac{\partial \bar{f}_{e}\left(v_{\|}^{\prime}, v_{\perp}^{\prime}, \mathbf{x}, t\right)}{\partial t}+\dot{\mathbf{x}} \cdot \frac{\partial \bar{f}_{e}}{\partial \mathbf{x}}+\dot{v}_{\|}^{\prime} \frac{\partial \bar{f}_{e}}{\partial v_{\|}^{\prime}}+\dot{v}_{\perp}^{\prime} \frac{\partial \bar{f}_{e}}{\partial v_{\perp}^{\prime}}=\left\langle C_{e e}\left[f_{e}, f_{e}\right]+C_{e \iota}\left[f_{e}, f_{\iota}\right]\right\rangle_{\alpha}
$$

where the collision operators on the right-hand-side are of order $\delta^{3}\left(v_{t h e} / L\right) f_{M e} \sim \delta_{e} \nu_{*}\left(v_{t h e} / L\right) f_{M e}$ and the coefficient functions $\dot{\mathbf{x}}, \dot{v}_{\|}^{\prime}$ and $\dot{v}_{\perp}^{\prime}$ of the collisionless streaming operator on the left-hand-side can be obtained by expanding the collisionless result of Ref.24 (which applies to the first order in $\delta_{e}$ ) for the present slow flow, neglecting terms of order $\delta^{4}\left(v_{t h e} / L\right) f_{M e} \sim \delta_{e}^{2}\left(v_{t h e} / L\right) f_{M e}$. Thus one gets 


$$
\begin{gathered}
\dot{\mathbf{x}}=\mathbf{u}_{e}-\mathbf{u}_{D e}+v_{\|}^{\prime} \mathbf{b}+\frac{v_{\perp}^{\prime 2}}{2} \nabla \times\left(\frac{\mathbf{b}}{\Omega_{c e}}\right)+\left(v_{\|}^{\prime 2}-\frac{v_{\perp}^{\prime 2}}{2}\right) \frac{\mathbf{b} \times \boldsymbol{\kappa}}{\Omega_{c e}}, \\
\dot{v}_{\|}^{\prime}=\frac{\mathbf{b} \cdot\left(\nabla \cdot \mathbf{P}_{e}^{C G L}-\mathbf{F}_{e}^{c o l l}\right)}{m_{e} n}-v_{\|}^{\prime} \mathbf{b} \cdot\left[(\mathbf{b} \cdot \nabla)\left(\mathbf{u}_{e}-\mathbf{u}_{D e}\right)\right]-\frac{v_{\perp}^{\prime 2}}{2} \mathbf{b} \cdot \nabla \ln B+\frac{v_{\|}^{\prime} v_{\perp}^{\prime 2}}{2} \nabla \cdot\left(\frac{\mathbf{b} \times \boldsymbol{\kappa}}{\Omega_{c e}}\right)
\end{gathered}
$$

and

$$
\dot{v}_{\perp}^{\prime}=\frac{v_{\perp}^{\prime}}{2}\left\{\mathbf{b} \cdot\left[(\mathbf{b} \cdot \nabla)\left(\mathbf{u}_{e}-\mathbf{u}_{D e}\right)\right]-\nabla \cdot\left(\mathbf{u}_{e}-\mathbf{u}_{D e}\right)+v_{\|}^{\prime} \mathbf{b} \cdot \nabla \ln B-v_{\|}^{\prime 2} \nabla \cdot\left(\frac{\mathbf{b} \times \boldsymbol{\kappa}}{\Omega_{c e}}\right)\right\}
$$

where

$$
\mathbf{u}_{D e}=\frac{1}{m_{e} n \Omega_{c e}}\left[\mathbf{b} \times \nabla p_{e \perp}+\left(p_{e \|}-p_{e \perp}\right)(\mathbf{b} \times \boldsymbol{\kappa})\right]
$$

is the electron diamagnetic drift velocity, $\boldsymbol{\kappa}=(\mathbf{b} \cdot \nabla) \mathbf{b}$ is the magnetic curvature and

$$
\mathbf{b} \cdot\left(\nabla \cdot \mathbf{P}_{e}^{C G L}\right)=\mathbf{b} \cdot \nabla p_{e \|}-\left(p_{e \|}-p_{e \perp}\right) \mathbf{b} \cdot \nabla \ln B
$$

The asymptotic expansion is carried on, after substituting Eq.(18) for $\bar{f}_{e}$ with $\bar{f}_{N M e}=O\left(\delta^{2} f_{M e}\right)+$ $O\left(\delta^{3} f_{M e}\right)$ and using the fluid equations (9) and (11) to eliminate the time derivative of the Maxwellian. In the lowest order, whereby $\delta^{2}\left(v_{t h e} / L\right) f_{M e}$ is negligible, Eqs.(19-24) along with (9) and (11) yield:

$$
v_{\|}^{\prime} \mathbf{b} \cdot \frac{\partial f_{M e}}{\partial \mathbf{x}}+\left[\frac{\mathbf{b} \cdot \nabla\left(n T_{e}\right)}{m_{e} n}-\frac{v_{\perp}^{\prime 2}}{2} \mathbf{b} \cdot \nabla \ln B\right] \frac{\partial f_{M e}}{\partial v_{\|}^{\prime}}+\frac{v_{\|}^{\prime} v_{\perp}^{\prime}}{2} \mathbf{b} \cdot \nabla \ln B \frac{\partial f_{M e}}{\partial v_{\perp}^{\prime}}=O\left(\delta^{2} \frac{v_{\text {the }}}{L} f_{M e}\right) .
$$

This means that the Maxwellian must be a solution of the collisionless, stationary, zero-Larmor-radius drift-kinetic equation, and this is guaranteed by the assumed small parallel temperature gradient ordering (5) $\mathbf{b} \cdot \nabla \ln T_{e}=O\left(\delta^{2}\right)$. Equation (25) does not impose any contraint on the parallel density gradient because its two terms proportional to $\mathbf{b} \cdot \nabla \ln n$, namely the one from the free-streaming operator and the one from the parallel acceleration due to the electric field piece proportional to the density gradient, cancel exactly. The terms proportional to $\mathbf{b} \cdot \nabla \ln B$ cancel too. Therefore, the expansion of the drift-kinetic equation can proceed as planned to the highest accuracy of order $\delta^{3}\left(v_{\text {the }} / L\right) f_{M e}$, assuming the maximal ordering for the parallel density gradient $\mathbf{b} \cdot \nabla \ln n=O(1)$. Changing to polar coordinates in velocity space $\left(v_{\|}^{\prime}=v^{\prime} \cos \chi, v_{\perp}^{\prime}=v^{\prime} \sin \chi\right)$, the final result is the following equation for $\bar{f}_{N M e}$ : 


$$
\begin{gathered}
\frac{\partial \bar{f}_{N M e}}{\partial t}+\cos \chi\left(v^{\prime} \mathbf{b} \cdot \frac{\partial \bar{f}_{N M e}}{\partial \mathbf{x}}+v_{t h e}^{2} \mathbf{b} \cdot \nabla \ln n \frac{\partial \bar{f}_{N M e}}{\partial v^{\prime}}\right)-\frac{\sin \chi}{v^{\prime}}\left(v_{t h e}^{2} \mathbf{b} \cdot \nabla \ln n-\frac{v^{\prime 2}}{2} \mathbf{b} \cdot \nabla \ln B\right) \frac{\partial \bar{f}_{N M e}}{\partial \chi}= \\
=\left\{\cos \chi \frac{v^{\prime}}{2 T_{e}}\left(5-\frac{v^{\prime 2}}{v_{\text {the }}^{2}}\right) \mathbf{b} \cdot \nabla T_{e}+\cos \chi \frac{v^{\prime}}{n T_{e}} \mathbf{b} \cdot\left[\frac{2}{3} \nabla\left(p_{e}-p_{e \perp}\right)-\left(p_{e \|}-p_{e \perp}\right) \nabla \ln B-\mathbf{F}_{e}^{c o l l}\right]+\right. \\
+P_{2}(\cos \chi) \frac{v^{\prime 2}}{3 v_{\text {the }}^{2}}\left(\nabla \cdot \mathbf{u}_{e}-3 \mathbf{b} \cdot\left[(\mathbf{b} \cdot \nabla) \mathbf{u}_{e}\right]\right)+\frac{1}{3 n T_{e}}\left(\frac{v^{\prime 2}}{v_{\text {the }}^{2}}-3\right)\left[\nabla \cdot\left(q_{e \|} \mathbf{b}\right)-G_{e}^{c o l l}\right]+ \\
+\frac{1}{6 e B}\left[2 P_{2}(\cos \chi) \frac{v^{\prime 2}}{v_{\text {the }}^{2}}\left(\frac{v^{\prime 2}}{v_{\text {the }}^{2}}-5\right)+\frac{v^{\prime 4}}{v_{\text {the }}^{4}}-10 \frac{v^{\prime 2}}{v_{\text {the }}^{2}}+15\right](\mathbf{b} \times \boldsymbol{\kappa}) \cdot \nabla T_{e}+ \\
+\frac{1}{6 e B}\left[-P_{2}(\cos \chi) \frac{v^{\prime 2}}{v_{\text {the }}^{2}}\left(\frac{v^{\prime 2}}{v_{\text {the }}^{2}}-5\right)+\frac{v^{\prime 4}}{v_{\text {the }}^{4}}-10 \frac{v^{\prime 2}}{v_{\text {the }}^{2}}+15\right](\mathbf{b} \times \nabla \ln B) \cdot \nabla T_{e}+ \\
\left.+P_{2}(\cos \chi) \frac{v^{\prime 2}}{3 e B v_{\text {the }}^{2}}(\mathbf{b} \times \nabla \ln n) \cdot \nabla T_{e}\right\} f_{M e}+\left\langle C_{e e}\left[f_{e}, f_{e}\right]+C_{e \iota}\left[f_{e}, f_{\iota}\right]\right\rangle_{\alpha}
\end{gathered}
$$

where $P_{l}$ denote the Legendre polynomials, i.e. $P_{2}(z)=3 z^{2} / 2-1 / 2$. Proceeding along the same lines to obtain the gyrophase-dependent part $\tilde{f}_{N M e}$ neglecting $O\left(\delta^{4} f_{M e}\right)$, the result is simply

$$
\tilde{f}_{N M e}=f_{M e} \frac{v^{\prime} \sin \chi}{2 e B v_{\text {the }}^{2}}\left(\frac{v^{\prime 2}}{v_{\text {the }}^{2}}-5\right)\left(\cos \alpha \mathbf{e}_{2}-\sin \alpha \mathbf{e}_{1}\right) \cdot \nabla T_{e} .
$$

The drift-kinetic equation (26) has some unconventional yet desirable features that are worth commenting on. First, it is referred to the moving-frame velocity coordinates $\left(v^{\prime}, \chi\right)$, which gives rise to the driving term proportional to $\nabla \cdot \mathbf{u}_{e}-3 \mathbf{b} \cdot\left[(\mathbf{b} \cdot \nabla) \mathbf{u}_{e}\right]$. In return, the fluid closure moments $(12-15)$ are evaluated directly without the need of a cumbersome substraction of the mean velocity. Second, the moving-frame derivation incorporates exactly the contribution of the electric field, consistent with the momentum conservation equation or generalized Ohm's law (10). Some pieces of the electric field are subject to cancellations, in particular the electron inertia piece is cancelled by an inertial force from the transformation to the moving frame. In addition, there is the above discussed cancellation 
of terms proportional to the parallel density gradient from free-streaming and parallel electric field acceleration on the Maxwellian. Equation (26) includes just the residual part of the electric field after these cancellations are taken into account. Finally, Eq.(26) is automatically consistent with the

required conditions that the $1, v_{\|}^{\prime}$ and $v^{\prime 2}$ velocity moments of $\bar{f}_{N M e}$ vanish because, under these conditions, the $1, v_{\|}^{\prime}$ and $v^{\prime 2}$ moments of Eq.(26) are exact identities.

The collision operators in Eq.(26) are needed only in their lowest non-vanishing order, $C_{e e}\left[f_{e}, f_{e}\right] \sim$ $C_{e \iota}\left[f_{e}, f_{\iota}\right] \sim \delta^{3}\left(v_{t h e} / L\right) f_{M e}$. Therefore, it is sufficient to use the following linearized forms:

$$
C_{e e}\left[f_{e}, f_{e}\right]=C_{e e}\left[f_{M e}, f_{N M e}\right]+C_{e e}\left[f_{N M e}, f_{M e}\right]
$$

and

$$
C_{e \iota}\left[f_{e}, f_{\iota}\right]=C_{e \iota}^{(3)}\left[f_{M e}, f_{\iota}\right]+C_{e \iota}^{(3)}\left[f_{N M e}, f_{M \iota}\right],
$$

where the superscripts indicate that only the leading parts of order $\delta^{3}\left(v_{t h e} / L\right) f_{M e}$ need to be retained. The details of these collision operators will be discussed in the next Section.

\section{Collision operators.}

The linearized collision operators $(28,29)$ will be taken in their complete Fokker-Planck-Landau form $^{22}$. In accordance with the present drift-kinetic derivation they must be expressed in the reference frame of the electron mean flow velocity, but this does not pose any difficulty by virtue of their Galilean invariance. Only some care has to be exercised to account for the different electron and ion mean velocities and to retain some electron-ion collision terms that produce leading-order effects as the result of contributions to the electron distribution function structure on the ion thermal velocity scale. For the sake of completeness, it is worth revisiting these collision operator expressions in detail.

The linearized electron-electron collision operator is completely standard and, by Galilean invariance, its laboratory frame expression applies equally to the moving reference frame. Thus, dropping the $(\mathbf{x}, t)$ arguments and with the electron collision frequency defined as

$$
\nu_{e} \equiv \frac{c^{4} e^{4} n \ln \Lambda_{e}}{4 \pi m_{e}^{2} v_{\text {the }}^{3}}
$$


in the rationalized electromagnetic system of units being used here, the Maxwellian-test part is the well known integral operator ${ }^{25,26}$ :

$$
C_{e e}\left[f_{M e}, f_{N M e}\right]\left(\mathbf{v}^{\prime}\right)=\frac{\nu_{e} v_{t h e}}{n} f_{M e}\left(v^{\prime}\right)\left\{4 \pi v_{t h e}^{2} f_{N M e}\left(\mathbf{v}^{\prime}\right)-\Phi\left[f_{N M e}\right]\left(\mathbf{v}^{\prime}\right)+v_{t h e}^{-2} \Xi\left[f_{N M e}\right]\left(\mathbf{v}^{\prime}\right)\right\}
$$

where $\Phi$ and $\Xi$ are the velocity space convolutions

$$
\Phi[f]\left(\mathbf{v}^{\prime}\right) \equiv \int d^{3} \mathbf{w} \frac{f(\mathbf{w})}{\left|\mathbf{v}^{\prime}-\mathbf{w}\right|}
$$

and

$$
\Xi[f]\left(\mathbf{v}^{\prime}\right) \equiv \frac{1}{2} \int d^{3} \mathbf{w} \frac{f(\mathbf{w})}{\left|\mathbf{v}^{\prime}-\mathbf{w}\right|^{3}}\left[\left|\mathbf{v}^{\prime}\right|^{2}|\mathbf{w}|^{2}-\left(\mathbf{v}^{\prime} \cdot \mathbf{w}\right)^{2}\right]
$$

or, in terms of Rosenbluth potentials,

$$
\frac{\partial}{\partial \mathbf{v}^{\prime}} \cdot \frac{\partial \Phi[f]\left(\mathbf{v}^{\prime}\right)}{\partial \mathbf{v}^{\prime}}=-4 \pi f\left(\mathbf{v}^{\prime}\right)
$$

and

$$
\Xi[f]\left(\mathbf{v}^{\prime}\right)=\left(\mathbf{v}^{\prime} \mathbf{v}^{\prime}\right): \frac{\partial^{2} \Psi[f]\left(\mathbf{v}^{\prime}\right)}{\partial \mathbf{v}^{\prime} \partial \mathbf{v}^{\prime}} \quad \text { with } \quad \frac{\partial}{\partial \mathbf{v}^{\prime}} \cdot \frac{\partial \Psi[f]\left(\mathbf{v}^{\prime}\right)}{\partial \mathbf{v}^{\prime}}=\Phi[f]\left(\mathbf{v}^{\prime}\right)
$$

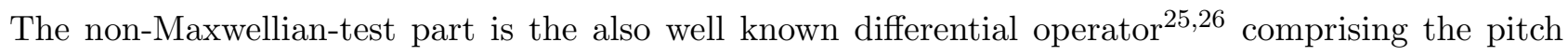
angle and energy scattering terms:

$$
\begin{gathered}
C_{e e}\left[f_{N M e}, f_{M e}\right]\left(\mathbf{v}^{\prime}\right)=\frac{\nu_{e} v_{\text {the }}^{3}}{v^{\prime 3}}\left\{\left[\varphi\left(\frac{v^{\prime}}{v_{\text {the }}}\right)-\xi\left(\frac{v^{\prime}}{v_{\text {the }}}\right)\right] \mathcal{L}\left[f_{N M e}\right]\left(\mathbf{v}^{\prime}\right)+\right. \\
\left.+\mathbf{v}^{\prime} \cdot \frac{\partial}{\partial \mathbf{v}^{\prime}}\left[\xi\left(\frac{v^{\prime}}{v_{\text {the }}}\right) \mathbf{v}^{\prime} \cdot \frac{\partial f_{N M e}\left(\mathbf{v}^{\prime}\right)}{\partial \mathbf{v}^{\prime}}+\frac{v^{\prime 2}}{v_{\text {the }}^{2}} \xi\left(\frac{v^{\prime}}{v_{\text {the }}}\right) f_{N M e}\left(\mathbf{v}^{\prime}\right)\right]\right\}
\end{gathered}
$$

where $\mathcal{L}$ is the Lorentz operator

$$
\mathcal{L}[f]\left(\mathbf{v}^{\prime}\right) \equiv \frac{v^{\prime 3}}{2} \frac{\partial}{\partial \mathbf{v}^{\prime}} \cdot\left\{\frac{1}{v^{\prime}} \frac{\partial f\left(\mathbf{v}^{\prime}\right)}{\partial \mathbf{v}^{\prime}}-\frac{1}{v^{\prime 3}}\left[\mathbf{v}^{\prime} \cdot \frac{\partial f\left(\mathbf{v}^{\prime}\right)}{\partial \mathbf{v}^{\prime}}\right] \mathbf{v}^{\prime}\right\}
$$

$\varphi$ is the error function

$$
\varphi\left(\frac{v^{\prime}}{v_{t h s}}\right)=\frac{v^{\prime}}{n} \Phi\left[f_{M s}\right]\left(v^{\prime}\right)=\frac{2}{(2 \pi)^{1 / 2}} \int_{0}^{v^{\prime} / v_{t h s}} d t \exp \left(-t^{2} / 2\right)
$$


and $\xi$ is the Chandrasekhar function

$$
\xi\left(\frac{v^{\prime}}{v_{t h s}}\right)=\frac{1}{n v^{\prime}} \Xi\left[f_{M s}\right]\left(v^{\prime}\right)=\frac{v_{t h s}^{2}}{v^{\prime 2}}\left[\varphi\left(\frac{v^{\prime}}{v_{t h s}}\right)-\frac{2 v^{\prime}}{(2 \pi)^{1 / 2} v_{t h s}} \exp \left(-\frac{v^{\prime 2}}{2 v_{t h s}^{2}}\right)\right]
$$

with the asymptotic behaviors: $\varphi(x \rightarrow 0)=2(2 \pi)^{-1 / 2} x, \quad \xi(x \rightarrow 0)=(2 / 3)(2 \pi)^{-1 / 2} x, \quad \varphi(x \rightarrow \infty)=1$ and $\xi(x \rightarrow \infty)=x^{-2}$.

Given that $f_{N M e} \sim \delta^{2} f_{M e}$ and $\nu_{e} \sim \delta v_{t h e} / L$, to obtain the non-Maxwellian-test part of the electron-ion collision operator with the required accuracy of $C_{e \iota}\left[f_{N M e}, f_{M \iota}\right] \sim \delta^{3}\left(v_{t h e} / L\right) f_{M e}$, one can neglect the mean flow difference between species $\left|\mathbf{u}_{\iota}-\mathbf{u}_{e}\right|=O\left(\delta v_{t h \iota}\right)$ in this term and take

$$
\begin{aligned}
& C_{e \iota}^{(3)}\left[f_{N M e}, f_{M \iota}\right]\left(\mathbf{v}^{\prime}\right)=\frac{\nu_{e} v_{t h e}^{3}}{v^{\prime 3}}\left\{\left[\varphi\left(\frac{v^{\prime}}{v_{t h \iota}}\right)-\xi\left(\frac{v^{\prime}}{v_{t h \iota}}\right)\right] \mathcal{L}\left[f_{N M e}\right]\left(\mathbf{v}^{\prime}\right)+\right. \\
& \left.+\mathbf{v}^{\prime} \cdot \frac{\partial}{\partial \mathbf{v}^{\prime}}\left[\xi\left(\frac{v^{\prime}}{v_{t h \iota}}\right) \mathbf{v}^{\prime} \cdot \frac{\partial f_{N M e}\left(\mathbf{v}^{\prime}\right)}{\partial \mathbf{v}^{\prime}}+\frac{m_{e} v^{\prime 2}}{m_{\iota} v_{t h \iota}^{2}} \xi\left(\frac{v^{\prime}}{v_{t h \iota}}\right) f_{N M e}\left(\mathbf{v}^{\prime}\right)\right]\right\} .
\end{aligned}
$$

Assuming $v^{\prime} \sim v_{t h e}$ and keeping only the leading contributions of order $\delta^{3}\left(v_{t h e} / L\right) f_{M e}$, the above reduces to

$$
C_{e \iota}^{(3)}\left[f_{N M e}, f_{M \iota}\right]\left(\mathbf{v}^{\prime} \sim v_{t h e}\right)=\frac{\nu_{e} v_{\text {the }}^{3}}{v^{3}} \mathcal{L}\left[f_{N M e}\right]\left(\mathbf{v}^{\prime}\right) .
$$

This asymptotic expression (41) applies in most of the electron phase space, but is singular for $v^{\prime} \rightarrow 0$ and must be regularized in the small phase space region where $v^{\prime}$ becomes comparable to $v_{t h \iota}$. The present work will use the complete operator (40) in order to preserve its regularity all its physical properties. The exact expression of the Maxwellian-test part of the linearized electron-ion collision operator is

$$
\begin{gathered}
C_{e \iota}\left[f_{M e}, f_{\iota}\right]\left(\mathbf{v}^{\prime}\right)=\frac{\nu_{e} v_{t h e}}{n} f_{M e}\left(v^{\prime}\right)\left\{\frac{4 \pi m_{e} v_{\text {the }}^{2}}{m_{\iota}} f_{\iota}\left(\mathbf{v}^{\prime}-\mathbf{u}_{\iota}+\mathbf{u}_{e}\right)-\Phi\left[f_{\iota}\right]\left(\mathbf{v}^{\prime}-\mathbf{u}_{\iota}+\mathbf{u}_{e}\right)+\right. \\
\left.+\left(\frac{m_{e}}{m_{\iota}}-1\right) \mathbf{v}^{\prime} \cdot \frac{\partial \Phi\left[f_{\iota}\right]\left(\mathbf{v}^{\prime}-\mathbf{u}_{\iota}+\mathbf{u}_{e}\right)}{\partial \mathbf{v}^{\prime}}+\frac{\mathbf{v}^{\prime} \mathbf{v}^{\prime}}{v_{\text {the }}^{2}}: \frac{\partial^{2} \Psi\left[f_{\iota}\right]\left(\mathbf{v}^{\prime}-\mathbf{u}_{\iota}+\mathbf{u}_{e}\right)}{\partial \mathbf{v}^{\prime} \partial \mathbf{v}^{\prime}}\right\}
\end{gathered}
$$

and, keeping its leading terms of order $\delta^{3}\left(v_{t h e} / L\right) f_{M e}$ for $v^{\prime} \sim v_{t h e}$, it reduces to

$$
C_{e \iota}^{(3)}\left[f_{M e}, f_{\iota}\right]\left(\mathbf{v}^{\prime} \sim v_{t h e}\right)=\nu_{e} v_{t h e} f_{M e}\left(v^{\prime}\right)\left[\frac{\mathbf{v}^{\prime} \cdot\left(\mathbf{u}_{\iota}-\mathbf{u}_{e}\right)}{v^{\prime 3}}+\frac{m_{e}}{m_{\iota} v^{\prime}}\left(\frac{T_{\iota}}{T_{e}}-1\right)\right] .
$$


Again, this asymptotic expression (43) applies in most of the electron phase space where $v^{\prime} \sim v_{\text {the }}$, but is singular for $v^{\prime} \rightarrow 0$ and so it must be regularized in the small phase space region where $v^{\prime}$ becomes comparable to $v_{t h \iota}$. It has the noteworthy feature that it does not depend on the non-Maxwellian part of the ion distribution function as a consequence of the small mass ratio and other assumed orderings. The present work will adopt a regularized form obtained by retaining just the lowest-order limit of Eq.(42) for $v^{\prime} \sim v_{t h \iota}$, which also requires only the ion Maxwellian part. The result, that matches (43) for $v^{\prime} \gg v_{t h \iota}$ and restores the particle conservation property lacking in (43), is

$$
\begin{gathered}
C_{e \iota}^{(3)}\left[f_{M e}, f_{\iota}\right]\left(\mathbf{v}^{\prime}\right)=\nu_{e} v_{t h e} f_{M e}\left(v^{\prime}\right)\left[\left(\frac{T_{e}}{T_{\iota}}-1\right) \frac{4 \pi v_{t h \iota}^{2}}{n} f_{M \iota}\left(v^{\prime}\right)+\right. \\
\left.+\frac{\mathbf{v}^{\prime} \cdot\left(\mathbf{u}_{\iota}-\mathbf{u}_{e}\right)}{v_{t h \iota}^{2} v^{\prime}} \xi\left(\frac{v^{\prime}}{v_{t h \iota}}\right)+\frac{m_{e}}{m_{\iota}}\left(\frac{T_{\iota}}{T_{e}}-1\right) \frac{v^{\prime}}{v_{t h \iota}^{2}} \xi\left(\frac{v^{\prime}}{v_{t h \iota}}\right)\right] .
\end{gathered}
$$

It is now straightforward to obtain the gyrophase averages of the four collision operator terms $(31,36,40,44)$ that enter in the drift-kinetic equation $(26)$. The homogeneous functional of $f_{N M e}$ that results from the gyro-average of the sum of Eqs.(31), (36) and (40) is readily verified to depend only on the gyro-averaged part of the distribution function, $\bar{f}_{N M e}$ :

$$
\left\langle C_{e e}\left[f_{M e}, f_{N M e}\right]+C_{e e}\left[f_{N M e}, f_{M e}\right]+C_{e \iota}^{(3)}\left[f_{N M e}, f_{M \iota}\right]\right\rangle_{\alpha}\left(v^{\prime}, \chi\right)=\mathcal{C}_{e}\left[\bar{f}_{N M e}\right]\left(v^{\prime}, \chi\right)
$$

Moreover, this operator is diagonal in a Legendre polynomial representation of the dependence on the angular variable $\chi$. Thus, for $f\left(v^{\prime}, \chi\right)=\sum_{l=0}^{\infty} f_{l}\left(v^{\prime}\right) P_{l}(\cos \chi)$,

$$
\mathcal{C}_{e}\left[\sum_{l=0}^{\infty} f_{l} P_{l}\right]\left(v^{\prime}, \chi\right)=\sum_{l=0}^{\infty} \mathcal{C}_{e, l}\left[f_{l}\right]\left(v^{\prime}\right) P_{l}(\cos \chi)
$$

where the one-dimensional operators $\mathcal{C}_{e, l}$ are:

$$
\begin{aligned}
& \mathcal{C}_{e, l}\left[f_{l}\right]\left(v^{\prime}\right)=\frac{\nu_{e} v_{t h e}}{n} f_{M e}\left(v^{\prime}\right)\left\{4 \pi v_{\text {the }}^{2} f_{l}\left(v^{\prime}\right)-\Phi_{l}\left[f_{l}\right]\left(v^{\prime}\right)+\frac{v^{\prime 2}}{v_{\text {the }}^{2}} \frac{d^{2} \Psi_{l}\left[f_{l}\right]\left(v^{\prime}\right)}{d v^{\prime 2}}\right\}+ \\
& +\frac{\nu_{e} v_{t h e}^{3}}{v^{\prime 2}} \frac{d}{d v^{\prime}}\left\{\xi\left(\frac{v^{\prime}}{v_{t h e}}\right)\left[v^{\prime} \frac{d f_{l}\left(v^{\prime}\right)}{d v^{\prime}}+\frac{v^{\prime 2}}{v_{\text {the }}^{2}} f_{l}\left(v^{\prime}\right)\right]+\xi\left(\frac{v^{\prime}}{v_{t h \iota}}\right)\left[v^{\prime} \frac{d f_{l}\left(v^{\prime}\right)}{d v^{\prime}}+\frac{m_{e} v^{\prime 2}}{m_{\iota} v_{t h \iota}^{2}} f_{l}\left(v^{\prime}\right)\right]\right\}-
\end{aligned}
$$

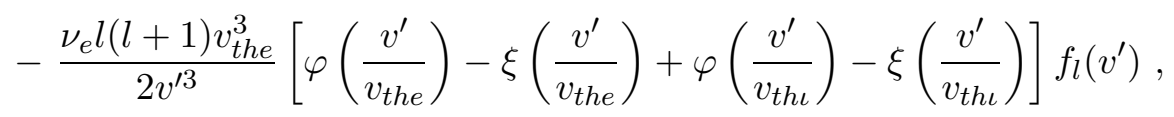


with

$$
\frac{1}{v^{\prime 2}} \frac{d}{d v^{\prime}}\left\{v^{\prime 2} \frac{d \Phi_{l}\left[f_{l}\right]\left(v^{\prime}\right)}{d v^{\prime}}\right\}-\frac{l(l+1)}{v^{\prime 2}} \Phi_{l}\left[f_{l}\right]\left(v^{\prime}\right)=-4 \pi f_{l}\left(v^{\prime}\right)
$$

and

$$
\frac{1}{v^{\prime 2}} \frac{d}{d v^{\prime}}\left\{v^{\prime 2} \frac{d \Psi_{l}\left[f_{l}\right]\left(v^{\prime}\right)}{d v^{\prime}}\right\}-\frac{l(l+1)}{v^{\prime 2}} \Psi_{l}\left[f_{l}\right]\left(v^{\prime}\right)=\Phi_{l}\left[f_{l}\right]\left(v^{\prime}\right)
$$

The inhomogeneous term that results from the gyro-average of Eq.(44) is

$$
\left\langle C_{e \iota}^{(3)}\left[f_{M e}, f_{\iota}\right]\right\rangle_{\alpha}\left(v^{\prime}, \chi\right)=\mathcal{D}_{e, 0}\left(v^{\prime}\right)+\mathcal{D}_{e, 1}\left(v^{\prime}\right) \cos \chi
$$

where

$$
\mathcal{D}_{e, 0}\left(v^{\prime}\right)=\nu_{e} v_{\text {the }} f_{M e}\left(v^{\prime}\right)\left[\left(\frac{T_{e}}{T_{\iota}}-1\right) \frac{4 \pi v_{t h \iota}^{2}}{n} f_{M \iota}\left(v^{\prime}\right)+\frac{m_{e}}{m_{\iota}}\left(\frac{T_{\iota}}{T_{e}}-1\right) \frac{v^{\prime}}{v_{t h \iota}^{2}} \xi\left(\frac{v^{\prime}}{v_{t h \iota}}\right)\right]
$$

and, in terms of the parallel current $j_{\|}=e n \mathbf{b} \cdot\left(\mathbf{u}_{\iota}-\mathbf{u}_{e}\right)$,

$$
\mathcal{D}_{e, 1}\left(v^{\prime}\right)=\nu_{e} f_{M e}\left(v^{\prime}\right) \frac{v_{t h e} j_{\|}}{v_{t h \iota}^{2} e n} \xi\left(\frac{v^{\prime}}{v_{t h \iota}}\right) .
$$

The collisional moments $(14,15)$ that appear in the fluid $(10,11)$ and drift-kinetic $(26)$ equations can now be evaluated. For these, the asymptotic form $(41,43)$ of the electron-ion collision operator for $v^{\prime} \sim v_{\text {the }}$ yields convergent integrals and is therefore sufficient. Using also the solution (27) for the gyrophase-dependent part of the distribution function, one gets

$$
\mathbf{F}_{e}^{\text {coll }}=\frac{2 m_{e} \nu_{e}}{3(2 \pi)^{1 / 2} e} \mathbf{j}-\frac{m_{e} \nu_{e} n}{(2 \pi)^{1 / 2} e B} \mathbf{b} \times \nabla T_{e}-2 \pi m_{e} \nu_{e} v_{t h e}^{3} \int_{0}^{\infty} d v^{\prime} \int_{0}^{\pi} d \chi \sin \chi \cos \chi \bar{f}_{N M e}\left(v^{\prime}, \chi\right) \mathbf{b}
$$

and

$$
G_{e}^{\text {coll }}=\frac{2 m_{e} \nu_{e} n}{(2 \pi)^{1 / 2} m_{\iota}}\left(T_{\iota}-T_{e}\right)
$$

Now, the only kinetic variables left to close the fluid system are the three scalars

$$
\left(p_{e \|}-p_{e \perp}\right)=2 \pi m_{e} \int_{0}^{\infty} d v^{\prime} v^{\prime 4} \int_{0}^{\pi} d \chi \sin \chi P_{2}(\cos \chi) \bar{f}_{N M e},
$$




$$
q_{e \|}=\pi m_{e} \int_{0}^{\infty} d v^{\prime} v^{\prime 5} \int_{0}^{\pi} d \chi \sin \chi \cos \chi \bar{f}_{N M e}
$$

and $F_{e \|}^{c o l l}$ given by the parallel component of Eq.(53). These involve only the gyro-averaged part of the distribution function in the moving frame of the macroscopic flow, $\bar{f}_{N M e}\left(v^{\prime}, \chi, \mathbf{x}, t\right)$, and can therefore be readily evaluated from the solution of the drift-kinetic equation (26).

\section{Application to an axisymmetric toroidal equilibrium.}

In developing the above closed model, the objective was a consistent formalism applicable to dynamical plasma processes (prime examples of which would be the sawtooth and "neoclassical tearing" instabilities in tokamaks), that would facilitate the compatibility between the fluid and drift-kinetic sides of the description. The adopted choices of a reference frame tied to the macroscopic fluid velocity and a lowest order Maxwellian distribution function whose argument is just the particle random kinetic energy were found particularly well suited for this purpose. It is nonetheless illustrative to show how such a formulation applies to the special case of an axisymmetric equilibrium, a problem that is studied traditionally in the framework of conventional neoclassical theory ${ }^{18-20}$, working in the laboratory reference frame with lowest order Maxwellians defined in terms of particle constants of motion.

In a time-independent system with axisymmetric toroidal geometry, where $\zeta$ denotes the ignorable azimuthal angle of the cylindrical coordinates $(R, \zeta, Z)$, the quasineutral Maxwell and continuity equations become

$$
\begin{gathered}
\nabla \cdot \mathbf{B}=0, \\
\mathbf{j}=e n\left(\mathbf{u}_{\iota}-\mathbf{u}_{e}\right)=\nabla \times \mathbf{B}, \\
\mathbf{E}=-\nabla \phi-V_{0} \nabla \zeta,
\end{gathered}
$$

and

$$
\nabla \cdot\left(n \mathbf{u}_{e}\right)=0
$$

Here, the electric potential has been split into the single-valued part $\phi$ and the multi-valued part $V_{0} \zeta$ associated with a constant loop voltage $2 \pi V_{0}$. Equations $(57,58)$, together with the axisymmetry 
condition, yield the standard representations for the magnetic field and the electric current:

$$
\begin{gathered}
\mathbf{B}=\nabla \psi \times \nabla \zeta+R B_{\zeta} \nabla \zeta, \\
\mathbf{j}=\nabla\left(R B_{\zeta}\right) \times \nabla \zeta-R^{2} \nabla \cdot\left(R^{-2} \nabla \psi\right) \nabla \zeta,
\end{gathered}
$$

and it will be assumed that a toroidally confined equilibrium solution with nested magnetic surfaces of constant $\psi$ exists. The stationary electron momentum and temperature fluid equations are:

$$
e n\left(\mathbf{E}+\mathbf{u}_{e} \times \mathbf{B}\right)+\nabla\left(n T_{e}\right)+\nabla \cdot\left[\left(p_{e \|}-p_{e \perp}\right)(\mathbf{b b}-\mathbf{I} / 3)\right]-\mathbf{F}_{e}^{\text {coll }}=0
$$

and

$$
\frac{3 n}{2} \mathbf{u}_{e} \cdot \nabla T_{e}+n T_{e} \nabla \cdot \mathbf{u}_{e}+\nabla \cdot\left(q_{e \|} \mathbf{b}-\frac{5 n T_{e}}{2 e B} \mathbf{b} \times \nabla T_{e}\right)-G_{e}^{\text {coll }}=0 .
$$

The only ion information to be needed, besides its Maxwellian temperature satisfying the small parallel gradient condition $\mathbf{b} \cdot \nabla T_{\iota}=O\left(\delta^{2} T_{\iota} / L\right)$, is the leading order momentum conservation equation

$$
-e n\left(\mathbf{E}+\mathbf{u}_{\iota} \times \mathbf{B}\right)+\nabla\left(n T_{\iota}\right)=O\left(\delta^{2} n T_{\iota} / L\right)
$$

Through its first order, i.e. neglecting $\delta^{2}$, the above stationary fluid system yields the well known relations ${ }^{2}$ :

$$
n=N^{(0)}(\psi), \quad T_{s}=T_{s}^{(0)}(\psi), \quad \phi=\phi^{(1)}(\psi)=O\left(T_{s} / e\right), \quad R B_{\zeta}=\left(R B_{\zeta}\right)^{(0)}(\psi) \equiv I(\psi),
$$

and

$$
\mathbf{u}_{s}=\mathbf{u}_{s}^{(1)}=U_{s}(\psi) \mathbf{B}+R^{2}\left[\frac{d \phi^{(1)}}{d \psi}+\frac{1}{e_{s} N^{(0)}} \frac{d\left(N^{(0)} T_{s}^{(0)}\right)}{d \psi}\right] \nabla \zeta=O\left(\delta v_{t h \iota}\right),
$$

with $d I(\psi) / d \psi=e N^{(0)}(\psi)\left[U_{\iota}(\psi)-U_{e}(\psi)\right]$. From these, it follows that

$$
\begin{gathered}
\nabla \psi \cdot(\mathbf{b} \times \boldsymbol{\kappa})=\nabla \psi \cdot(\mathbf{b} \times \nabla \ln B)=I(\psi) \mathbf{b} \cdot \nabla \ln B \\
\nabla \cdot \mathbf{u}_{s}=0 \quad \text { and } \quad \mathbf{b} \cdot\left[(\mathbf{b} \cdot \nabla) \mathbf{u}_{s}\right]=U_{s}(\psi) \mathbf{b} \cdot \nabla \ln B .
\end{gathered}
$$


Proceeding now to the highest retained accuracy of $O\left(\delta^{3} n m_{e} v_{\text {the }}^{2} / L\right)$, the parallel component of the equilibrium electron momentum equation (63) yields

$$
N^{(0)} T_{e}^{(0)} \mathbf{b} \cdot \nabla\left(\frac{e \phi}{T_{e}^{(0)}}-\frac{n}{N^{(0)}}-\frac{T_{e}}{T_{e}^{(0)}}\right)=\mathbf{b} \cdot\left[\frac{2}{3} \nabla\left(p_{e \|}-p_{e \perp}\right)-\left(p_{e \|}-p_{e \perp}\right) \nabla \ln B-\mathbf{F}_{e}^{c o l l}\right]-\frac{e V_{0} N^{(0)} I}{B R^{2}}
$$

and, to the highest retained accuracy of $O\left(\delta^{3} n m_{e} v_{t h e}^{3} / L\right)$, the equilibrium electron temperature equation (64) yields

$$
\nabla \cdot\left(q_{e \|} \mathbf{b}-\frac{5 N^{(0)} T_{e}^{(0)}}{2 e B} \mathbf{b} \times \nabla T_{e}^{(0)}\right)=\mathbf{B} \cdot \nabla\left(\frac{q_{e} \|}{B}+\frac{5 N^{(0)} T_{e}^{(0)} I}{2 e B^{2}} \frac{d T_{e}^{(0)}}{d \psi}\right)=G_{e}^{\text {coll }} .
$$

Note that, within this highest available accuracy, the equilibrium electron temperature equation provides just a relation between the components of the heat flux tangential to the magnetic surface, but does not provide any information on the higher order temperature correction $T_{e}(\mathbf{x})-T_{e}^{(0)}(\psi)$. Equation (71) has the solubility condition $\oint_{\psi} d l G_{e}^{\text {coll }} / B=0$ which, recalling (54), requires near equal electron and ion temperatures: $T_{e}^{(0)}(\psi)=T_{\iota}^{(0)}(\psi)$. Then it can be integrated to determine the parallel heat flux, up to a new free magnetic surface function:

$$
q_{e \|}=-\frac{5 N^{(0)} T_{e}^{(0)} I}{2 e B} \frac{d T_{e}^{(0)}}{d \psi}+Q_{e}(\psi) B
$$

Substituting the previous results in Eq.(26), the following axisymmetric equilibrium form of the electron drift-kinetic equation is obtained:

$$
\begin{gathered}
v^{\prime}\left(\cos \chi \mathbf{b} \cdot \frac{\partial \bar{f}_{N M e}}{\partial \mathbf{x}}+\frac{1}{2} \mathbf{b} \cdot \nabla \ln B \sin \chi \frac{\partial \bar{f}_{N M e}}{\partial \chi}\right)-\mathcal{C}_{e}\left[\bar{f}_{N M e}\right]= \\
=-f_{M e}^{(0)}\left\{P_{2}(\cos \chi) \frac{m_{e} v^{\prime 2}}{T_{e}^{(0)}} U_{e} B+\left[2+P_{2}(\cos \chi)\right] \frac{m_{e} v^{\prime 2}}{3 T_{e}^{(0)}}\left(\frac{5}{2}-\frac{m_{e} v^{\prime 2}}{2 T_{e}^{(0)}}\right) \frac{I}{e B} \frac{d T_{e}^{(0)}}{d \psi}\right\} \mathbf{b} \cdot \nabla \ln B+ \\
+\left\{f_{M e}^{(0)}\left[\mathbf{b} \cdot \nabla\left(\frac{e \phi}{T_{e}^{(0)}}-\frac{n}{N^{(0)}}\right)+\left(\frac{3}{2}-\frac{m_{e} v^{\prime 2}}{2 T_{e}^{(0)}}\right) \mathbf{b} \cdot \nabla\left(\frac{T_{e}}{T_{e}^{(0)}}\right)+\frac{e V_{0} I}{T_{e}^{(0)} B R^{2}}\right] v^{\prime}+\mathcal{D}_{e, 1}\right\} \cos \chi \cdot(
\end{gathered}
$$

Here, $f_{M e}^{(0)}$ denotes the Maxwellian with lowest order density and temperature, $N^{(0)}(\psi)$ and $T_{e}^{(0)}(\psi)$. 
Similarly, the collisional terms $\mathcal{C}_{e}$ and $\mathcal{D}_{e, 1}$ require only these lowest order, magnetic surface function forms of the density and temperature. Neglecting the collisions and the loop voltage, i.e. accurate to $O\left(\delta^{2} f_{M e}\right)$, a particular solution of Eq. (73) is $\bar{f}_{N M e}^{(2)}=\left(g_{e, 0}+g_{e, 1} \cos \chi\right) f_{M e}^{(0)}$ where

$$
g_{e, 0}=\frac{e\left(\phi-\phi^{(1)}\right)}{T_{e}^{(0)}}-\frac{n-N^{(0)}}{N^{(0)}}-\left(\frac{m_{e} v^{\prime 2}}{T_{e}^{(0)}}-3\right) \frac{T_{e}-T_{e}^{(0)}}{2 T_{e}^{(0)}}
$$

represents a perturbative redefinition of the Maxwellian and

$$
g_{e, 1}=-\left[\frac{m_{e} U_{e} B}{T_{e}^{(0)}}+\frac{m_{e} I}{2 e B T_{e}^{(0)}}\left(\frac{m_{e} v^{\prime 2}}{T_{e}^{(0)}}-5\right) \frac{d T_{e}^{(0)}}{d \psi}\right] v^{\prime} .
$$

Thus, calling

$$
\bar{f}_{N M e}=h_{e}+\left(g_{e, 0}+g_{e, 1} \cos \chi\right) f_{M e}^{(0)},
$$

and substituting the explicit expression for $\mathcal{D}_{e, 1}(52)$, the equilibrium drift-kinetic equation reduces to

$$
\begin{gathered}
v^{\prime}\left(\cos \chi \mathbf{b} \cdot \frac{\partial h_{e}}{\partial \mathbf{x}}+\frac{1}{2} \mathbf{b} \cdot \nabla \ln B \sin \chi \frac{\partial h_{e}}{\partial \chi}\right)-\mathcal{C}_{e}\left[h_{e}\right]= \\
=\mathcal{C}_{e}\left[\left(g_{e, 0}+g_{e, 1} \cos \chi\right) f_{M e}^{(0)}\right]+\left[\frac{e V_{0} I}{T_{e}^{(0)} B R^{2}} v^{\prime}+\frac{\nu_{e} v_{t h e} j_{\|}}{v_{t h \iota}^{2} e N^{(0)}} \xi\left(\frac{v^{\prime}}{v_{t h \iota}}\right)\right] f_{M e}^{(0)} \cos \chi
\end{gathered}
$$

The action of the collision operator $\mathcal{C}_{e}(46-49)$ on the particular solution $\left(g_{e, 0}+g_{e, 1} \cos \chi\right) f_{M e}^{(0)}$ can be carried out analytically. Thus, within the maximum retained accuracy of order $\delta^{3}\left(v_{t h e} / L\right) f_{M e}$, $\mathcal{C}_{e}\left[g_{e, 0} f_{M e}^{(0)}\right]=0$ and

$$
\begin{gathered}
\mathcal{C}_{e}\left[g_{e, 1} \cos \chi f_{M e}^{(0)}\right]=\nu_{e} v_{t h e}\left\{\frac{U_{e} B}{v_{t h \iota}^{2}} \xi\left(\frac{v^{\prime}}{v_{t h \iota}}\right)+\right. \\
\left.+\frac{m_{e} I}{e B T_{e}^{(0)}} \frac{d T_{e}^{(0)}}{d \psi}\left[2 \varphi\left(\frac{v^{\prime}}{v_{t h e}}\right)-10 \xi\left(\frac{v^{\prime}}{v_{t h e}}\right)+\frac{1}{2} \varphi\left(\frac{v^{\prime}}{v_{t h \iota}}\right)-\frac{5 v_{t h e}^{2}}{2 v_{t h \iota}^{2}} \xi\left(\frac{v^{\prime}}{v_{t h \iota}}\right)\right]\right\} f_{M e}^{(0)} \cos \chi .
\end{gathered}
$$

Now, calling the right-hand-side of Eq.(77) $\mathcal{S}_{e} v^{\prime} \cos \chi$ and collecting its terms,

$$
\begin{gathered}
\mathcal{S}_{e}=\left\{\frac{e V_{0} I}{T_{e}^{(0)} B R^{2}}+\nu_{e}\left(\frac{j_{\|}}{e N^{(0)}}+U_{e} B\right) \frac{v_{t h e}}{v_{t h \iota}^{2} v^{\prime}} \xi\left(\frac{v^{\prime}}{v_{t h \iota}}\right)+\right. \\
\left.+\frac{\nu_{e} m_{e} I}{e B T_{e}^{(0)}} \frac{d T_{e}^{(0)}}{d \psi} \frac{v_{\text {the }}}{v^{\prime}}\left[2 \varphi\left(\frac{v^{\prime}}{v_{\text {the }}}\right)-10 \xi\left(\frac{v^{\prime}}{v_{t h e}}\right)+\frac{1}{2} \varphi\left(\frac{v^{\prime}}{v_{t h \iota}}\right)-\frac{5 v_{t h e}^{2}}{2 v_{t h \iota}^{2}} \xi\left(\frac{v^{\prime}}{v_{t h \iota}}\right)\right]\right\} f_{M e}^{(0)} .
\end{gathered}
$$


From the first order flow solution (67), the parallel current satisfies

$$
\frac{j_{\|}}{e N^{(0)}}=\mathbf{b} \cdot\left(\mathbf{u}_{\iota}-\mathbf{u}_{e}\right)=\left(U_{\iota}-U_{e}\right) B+\frac{I}{e N^{(0)} B} \frac{d P^{(0)}}{d \psi}
$$

with $P^{(0)}=N^{(0)}\left(T_{\iota}^{(0)}+T_{e}^{(0)}\right)$. Hence

$$
\begin{aligned}
& \mathcal{S}_{e}=\left\{\frac{e V_{0} I}{T_{e}^{(0)} B R^{2}}+\nu_{e}\left(U_{\iota} B+\frac{I}{e N^{(0)} B} \frac{d P^{(0)}}{d \psi}\right) \frac{v_{t h e}}{v_{t h \iota}^{2} v^{\prime}} \xi\left(\frac{v^{\prime}}{v_{t h \iota}}\right)+\right. \\
& \left.+\frac{\nu_{e} m_{e} I}{e B T_{e}^{(0)}} \frac{d T_{e}^{(0)}}{d \psi} \frac{v_{\text {the }}}{v^{\prime}}\left[2 \varphi\left(\frac{v^{\prime}}{v_{\text {the }}}\right)-10 \xi\left(\frac{v^{\prime}}{v_{t h e}}\right)+\frac{1}{2} \varphi\left(\frac{v^{\prime}}{v_{t h \iota}}\right)-\frac{5 v_{t h e}^{2}}{2 v_{t h \iota}^{2}} \xi\left(\frac{v^{\prime}}{v_{t h \iota}}\right)\right]\right\} f_{M e}^{(0)} .
\end{aligned}
$$

The last step is to use magnetic surface coordinates $(\psi, \theta)$ in the meridian plane $(R, Z)$ and to change variables in phase space from $\left(R, Z, v^{\prime}, \chi\right)$ to $\left(\psi, \theta, v^{\prime}, \lambda\right)$, with $\lambda(\psi, \theta, \chi)=\sin ^{2} \chi B_{\max }(\psi) / B(\psi, \theta)$ and $B_{\max }(\psi)$ equal to the maximum of $B$ on the $\psi$-magnetic surface (assuming no multiple relative maxima). Then, Eq.(77) becomes

$$
v_{\|}^{\prime}(\mathbf{b} \cdot \nabla \theta) \frac{\partial h_{e}}{\partial \theta}-\mathcal{C}_{e}\left[h_{e}\right]=\mathcal{S}_{e} v_{\|}^{\prime}
$$

where now the derivative with respect to $\theta$ is taken at constant $\psi, v^{\prime}$ and $\lambda$, and $v_{\|}^{\prime}$ signifies the function

$$
v_{\|}^{\prime}\left(\psi, \theta, v^{\prime}, \lambda\right)= \pm v^{\prime}\left[1-\lambda B(\psi, \theta) / B_{\max }(\psi)\right]^{1 / 2}
$$

Equation (82) has the same form as the conventional neoclassical banana equilibrium equation and can be solved using the same techniques. However, it has a somewhat different meaning in that its velocity space arguments are the components of the random velocity relative to the macroscopic flow and that its solution must be such that the $1, v_{\|}^{\prime}$ and $v^{\prime 2}$ moments of the complete non-Maxwellian part of the distribution function, $\bar{f}_{N M e}=h_{e}+\left(g_{e, 0}+g_{e, 1} \cos \chi\right) f_{M e}^{(0)}$, are equal to zero. This is compensated by the differences in the source function $\mathcal{S}_{e}$, leading to the same physical results as will be discussed next. As a bonus, this exercise will shed new light on some properties of the neoclassical equilibrium in the banana regime. 
Following the standard solution method of neoclassical theory ${ }^{18-20}$, one gets the perturbative solution of Eq.(82):

$$
h_{e}=\varsigma\left(v_{\|}^{\prime}\right) H(1-\lambda) K_{e}\left(\psi, v^{\prime}, \lambda\right)+h_{e}^{(3)}\left(\psi, \theta, v^{\prime}, \lambda\right)=O\left(\delta^{2} f_{M e}\right)+O\left(\delta^{3} f_{M e}\right)
$$

where $\varsigma\left(v_{\|}^{\prime}\right)=\operatorname{sign}\left(v_{\|}^{\prime}\right)= \pm 1, \quad H$ is the Heaviside step function and $h_{e}^{(3)}\left(\psi, \theta, v^{\prime}, \lambda\right)=O\left(\delta^{3} f_{M e}\right)$ is a function, even with respect to $v_{\|}^{\prime}$, that satisfies

$$
v_{\|}^{\prime}(\mathbf{b} \cdot \nabla \theta) \frac{\partial h_{e}^{(3)}}{\partial \theta}-\mathcal{C}_{e}\left[\varsigma H K_{e}\right]=\mathcal{S}_{e} v_{\|}^{\prime} .
$$

This has a solubility condition that serves to determine $K_{e}\left(\psi, v^{\prime}, \lambda\right)$ :

$$
\oint_{\psi, v^{\prime}, \lambda} d l v_{\|}^{\prime-1} \mathcal{C}_{e}\left[\varsigma H K_{e}\right]=-\oint_{\psi, v^{\prime}, \lambda} d l \mathcal{S}_{e}
$$

where the contour integral $\oint_{\psi, v^{\prime}, \lambda} d l$ along the magnetic field line at constant $\left(\psi, v^{\prime}, \lambda\right)$, is to be taken around one complete poloidal turn for $\lambda<1$ (passing domain); for $\lambda>1$ (trapped domain) the contour is a bounce back and forth between the two accessible zeros of $v_{\|}^{\prime}\left(\psi, \theta, v^{\prime}, \lambda\right)$, one segment on each of the two values of $\varsigma\left(v_{\|}^{\prime}\right)$. Since both $v_{\|}^{\prime-1} \mathcal{C}_{e}\left[\varsigma H K_{e}\right]$ and $\mathcal{S}_{e}$ are even functions with respect to $v_{\|}^{\prime}$, Eq.(86) is satisfied trivially for $\lambda>1$ and only the $\lambda<1$ domain needs consideration. The consistency of this perturbative solution requires a sufficiently smooth $h_{e}(84)$, such that it does not give rise to singularities when acted on by the collision operator $\mathcal{C}_{e}$, and this imposes the boundary conditions $K_{e}\left(\psi, v^{\prime}, 1\right)=0$ and $\partial K_{e}\left(\psi, v^{\prime}, 1\right) / \partial \lambda=0$. If a solution to (86) satisfying these boundary conditions cannot be found, then the original Eq.(82) must be taken into account, either globally or in a boundary layer near $\lambda=1$.

Provided a satisfactory solution for $h_{e}$ of the form (84) exists, then, recalling $(75,76)$, the part of the distribution function that is odd with respect to $v_{\|}^{\prime}$ is:

$$
\bar{f}_{N M e}^{o d d}=\varsigma\left(v_{\|}^{\prime}\right) H(1-\lambda) K_{e}\left(\psi, v^{\prime}, \lambda\right)-v_{\|}^{\prime}\left[\frac{m_{e} U_{e} B}{T_{e}^{(0)}}+\frac{m_{e} I}{2 e B T_{e}^{(0)}}\left(\frac{m_{e} v^{\prime 2}}{T_{e}^{(0)}}-5\right) \frac{d T_{e}^{(0)}}{d \psi}\right] f_{M e}^{(0)}=O\left(\delta^{2} f_{M e}\right) .
$$

This must satisfy $\int d^{3} \mathbf{v}^{\prime} v_{\|}^{\prime} \bar{f}_{N M e}^{o d d}=0$, which yields the following solution for the parallel flow stream function:

$$
U_{e}(\psi)=\frac{2 \pi}{N^{(0)}(\psi) B_{\max }(\psi)} \int_{0}^{\infty} d v^{\prime} v^{\prime 3} \int_{0}^{1} d \lambda K_{e}\left(\psi, v^{\prime}, \lambda\right)
$$


Given the structure of the source function $\mathcal{S}_{e}(81)$, one can see that $U_{e}$ will be made of terms proportional to $V_{0} / \nu_{e}, U_{\iota}, d P^{(0)} / d \psi$ and $d T_{e}^{(0)} / d \psi$, corresponding to electron flow contributions to the Ohmic and bootstrap parts of the electric current ${ }^{27}$. Besides, knowledge of $\bar{f}_{N M e}^{o d d}$ allows one to evaluate the odd closures $(53,56)$ :

$$
F_{e \|}^{c o l l}=\frac{2 m_{e} \nu_{e}}{3(2 \pi)^{1 / 2}}\left(\frac{j_{\|}}{e}+N^{(0)} U_{e} B-\frac{3 N^{(0)} I}{2 e B} \frac{d T_{e}^{(0)}}{d \psi}\right)-\frac{2 \pi m_{e} \nu_{e} v_{t h e}^{3} B}{B_{\max }} \int_{0}^{\infty} d v^{\prime} \int_{0}^{1} d \lambda K_{e}\left(\psi, v^{\prime}, \lambda\right)
$$

and

$$
q_{e \|}=-\frac{5 N^{(0)} T_{e}^{(0)} I}{2 e B} \frac{d T_{e}^{(0)}}{d \psi}+\frac{\pi T_{e}^{(0)} B}{B_{\max }} \int_{0}^{\infty} d v^{\prime} v^{\prime 3}\left(\frac{m_{e} v^{\prime 2}}{T_{e}^{(0)}}-5\right) \int_{0}^{1} d \lambda K_{e}\left(\psi, v^{\prime}, \lambda\right),
$$

the latter being consistent with the fluid result (72) and serving to specify the magnetic surface function $Q_{e}(\psi)$.

Recalling now $(74,76)$, the part of the distribution function that is even with respect to $v_{\|}^{\prime}$ is

$$
\bar{f}_{N M e}^{e v e n}=h_{e}^{(3)}\left(\psi, \theta, v^{\prime}, \lambda\right)+\left[\frac{e\left(\phi-\phi^{(1)}\right)}{T_{e}^{(0)}}-\frac{n-N^{(0)}}{N^{(0)}}-\left(\frac{m_{e} v^{\prime 2}}{T_{e}^{(0)}}-3\right) \frac{T_{e}-T_{e}^{(0)}}{2 T_{e}^{(0)}}\right] f_{M e}^{(0)} .
$$

This must satisfy $\int d^{3} \mathbf{v}^{\prime} \bar{f}_{N M e}^{e v e n}=0$ and $\int d^{3} \mathbf{v}^{\prime} v^{\prime 2} \bar{f}_{N M e}^{e v e n}=0$, which yield

$$
\frac{e\left(\phi-\phi^{(1)}\right)}{T_{e}^{(0)}}-\frac{n-N^{(0)}}{N^{(0)}}=-\frac{2 \pi B}{N^{(0)} B_{\max }} \int_{0}^{\infty} d v^{\prime} v^{\prime 2} \int_{0}^{B_{\max } / B} \frac{d \lambda}{\left(1-\lambda B / B_{\max }\right)^{1 / 2}} h_{e}^{(3)}=O\left(\delta^{3}\right)
$$

and

$$
\frac{T_{e}-T_{e}^{(0)}}{T_{e}^{(0)}}=\frac{2 \pi B}{3 N^{(0)} B_{\max }} \int_{0}^{\infty} d v^{\prime} v^{\prime 2}\left(\frac{m_{e} v^{\prime 2}}{T_{e}^{(0)}}-3\right) \int_{0}^{B_{\max } / B} \frac{d \lambda}{\left(1-\lambda B / B_{\max }\right)^{1 / 2}} h_{e}^{(3)}=O\left(\delta^{3}\right)
$$

The function $\bar{f}_{N M e}^{e v e n}$ would also specify the last remaining closure variable $\left(p_{e \|}-p_{e \perp}\right)(55)$ as

$$
\left(p_{e \|}-p_{e \perp}\right)=\frac{\pi m_{e} B}{B_{\max }} \int_{0}^{\infty} d v^{\prime} v^{4} \int_{0}^{B_{\max } / B} d \lambda \frac{2-3 \lambda B / B_{\max }}{\left(1-\lambda B / B_{\max }\right)^{1 / 2}} h_{e}^{(3)}=O\left(\delta^{3} N^{(0)} T_{e}^{(0)}\right) .
$$

So, the variables $\left(T_{e}-T_{e}^{(0)}\right) / T_{e}^{(0)}$ and $\left(p_{e \|}-p_{e \perp}\right) /\left(N^{(0)} T_{e}^{(0)}\right)$ that were originally ordered as $O\left(\delta^{2}\right)$ in 
the general formulation, are actually third order in the special case of this axisymmetric equilibrium. Much like the kinetic parallel heat flux solution (90) satisfies identically the fluid temperature equation, Eqs.(92-94) satisfy identically the fluid parallel momentum equation (70) if $h_{e}^{(3)}$ satisfies (85). Therefore, the equilibrium parallel Ohm's law (70) does not add any new information to the kinetic results (92-94) on the even part of the distribution function. Unfortunatelly, the kinetic equation (85) for the required function $h_{e}^{(3)}\left(\psi, \theta, v^{\prime}, \lambda\right)$ leaves unspecified a homogeneous solution of the form $H_{e}^{(3)}\left(\psi, v^{\prime}, \lambda\right)$ that would only be determined by a solubility condition in the next (fourth) order of the expansion. This goes beyond the accuracy retained in the present analysis or in the conventional low collisionality neoclassical theory (among other things, it would necessitate extending the drift-kinetic equation to the second order in the electron gyroradius and the collision operators to include the nonMaxwellian quadratic terms). One must therefore conclude that the considered perturbative solution of the low collisionality axisymmetric equilibrium system, which again is equivalent to the neoclassical solution in the electron low collisionality regime, does not determine the lowest significant orders of $\left(T_{e}-T_{e}^{(0)}\right) \sim \delta^{3} T_{e}^{(0)}$ or $\left(p_{e \|}-p_{e \perp}\right) \sim \delta^{3} N^{(0)} T_{e}^{(0)}$. Similarly, Eq.(92) implies that the poloidally varying part of the plasma electric potential that specifies the parallel electric field, $\phi(\psi, \theta)-\phi^{(1)}(\psi)$, can only be known in its second order adiabatic approximation:

$$
\frac{e\left(\phi-\phi^{(1)}\right)}{T_{e}^{(0)}}=\frac{n-N^{(0)}}{N^{(0)}}+O\left(\delta^{3}\right),
$$

where $n-N^{(0)}$ will come from a solution of the coupled ion system and its knowledge is granted here. This is consistent with the parallel Ohm's law (70), where it can be seen that the third order contribution to the parallel electric field due to the collisional friction force $F_{e \|}^{c o l l}$ is known after $(88,89)$, but there are unknown third order contributions from the parallel temperature gradient $\mathbf{b} \cdot \nabla\left(N^{(0)} T_{e}\right)$ and the parallel divergence of the anisotropic stress $\mathbf{b} \cdot\left[\frac{2}{3} \nabla\left(p_{e \|}-p_{e \perp}\right)-\left(p_{e \|}-p_{e \perp}\right) \nabla \ln B\right]$. One can take the magnetic surface average of (70) to annihilate its left-hand-side and obtain the following expression for the magnetic surface average of the parallel divergence of the anisotropic stress:

$$
\oint_{\psi} d l \mathbf{b} \cdot\left[\frac{2}{3} \nabla\left(p_{e \|}-p_{e \perp}\right)-\left(p_{e \|}-p_{e \perp}\right) \nabla \ln B\right]=\oint_{\psi} d l\left(F_{e \|}^{c o l l}+\frac{e V_{0} N^{(0)} I}{B R^{2}}\right) .
$$

This result, with (89) substituted for $F_{e \|}^{c o l l}$, is usually referred to as the averaged neoclassical parallel viscosity $^{28,29}$ but it does not provide any information on the parallel electric field. The third order parallel electric field, with its terms related to both the collisional friction force and the anisotropic stress, 
is crucial in processes involving magnetic reconnection such as the sawtooth and "neoclassical tearing" instabilities. Of course, these are three-dimensional dynamical problems for which the axisymmetric stationary analysis does not apply and the contributions to the parallel electric field are to be obtained unambiguously from an initial value (or perhaps normal mode) solution of the time-dependent equations given in Secs. III and IV. Anyway, the results of this Section should caution against the use of simplified formulas based on axisymmetric equilibrium considerations when studying such instabilities.

\section{Summary.}

A systematic expansion in uniform powers of $\rho_{\iota} / L \sim \nu_{*} \sim\left(m_{e} / m_{\iota}\right)^{1 / 2} \ll 1$ has yielded a consistently closed electron model, applicable to slow macroscopic phenomena in low collisionality, magnetically confined plasmas. The result is a fluid and drift-kinetic hybrid system. The fluid side includes the quasineutral Maxwell equations (7,8), the continuity equation (9) and the momentum conservation and temperature equations $(10,11)$, with explicit analytic expressions for the perpendicular components of the collisional friction force (53) and the collisional heat source (54). Three scalars are left as kinetic closure variables, namely the parallel collisional friction force (53) the pressure anisotropy (55) and the parallel heat flux (56). These involve moments of the gyrophase-averaged, non-Maxwellian part of the distribution function in the moving reference frame of the macroscopic electron flow that satisfies the drift-kinetic equation (26).

The form of the finite-Larmor-radius drift-kinetic equation (26) for the non-Maxwellian perturbation of the electron distribution function is one of the main results of this work. It refers to the random velocity variables in the moving frame of the macroscopic flow, thus allowing a direct evaluation of the fluid closure moments. Its derivation takes precisely into account the electric field and the time derivative of the Maxwellian, consistent with the simultaneous fluid equations. This drift-kinetic equation is also automatically consistent with the condition that the $1, v_{\|}^{\prime}$ and $v^{\prime 2}$ moments of the non-Maxwellian part of the distribution function vanish. Because of the adopted low collisionality and close to Maxwellian orderings, the gyrophase-averaged collision operators entering the drift-kinetic equation can be taken in their linearized version. Otherwise, full Fokker-Planck-Landau expressions 
are used. These result in the homogeneous integro-differential functional given by Eqs.(45-49) that includes Rosenbluth potentials of the non-Maxwellian electron field and pitch angle and energy scattering between electrons and between electron and ions, and the explicit inhomogeneous term given by Eqs.(50-52) that includes temperature equilibration and flow difference friction between electrons and ions.

As an illustrative application, the special limit of an axisymmetric toroidal equilibrium has been studied. This exercise shows how the present theory can recover the neoclassical banana regime results for the odd closures and the electron flow contribution to the bootstrap current. On the other hand it is shown that, in the special case of this axisymmetric equilibrium and unlike in a time-dependent system, the lowest significant orders of the pressure anisotropy and the temperature variation on a magnetic surface cannot be determined by the present theory or its equivalent neoclassical equilibrium theory in the low collisionality regime. Thus, the poloidally varying part of the plasma electric potential that specifies the parallel component of the stationary and axisymmetric electric field can only be known in the adiabatic approximation (95).

This work can be easily generalized to include an externally applied radio-frequency (RF) source, provided its effect after averaging over high frequency fields can be modeled by an additive term in the electron kinetic equation ${ }^{30}$. This would be the case for applied wave frequencies in the electroncyclotron range and the specifics of this generalization are given in an Appendix.

\section{Acknowledgements.}

The author acknowledges very useful discussions with J. Callen, P. Catto, C. Hegna and F. ParraDiaz. This work was sponsored by the U.S. Department of Energy under Grants Nos. DEFG0291ER54109, DEFC02-08ER54969 and DEFC02-06ER54855 at the Massachusetts Institute of Technology and as part of the author's participation in the Centers for Extended MHD Modeling (CEMM) and for Simulation of Wave Interactions with MHD (CSWIM). 


\section{Appendix: Generalization to include an RF source.}

A straightforward generalization of the present theory to include externally applied RF electromagnetic waves is possible, if such a source can be represented by an additive term in the electron kinetic equation after averaging over the high frequency fields. This would be feasible for applied wave frequencies in the electron-cyclotron range and has important applications in the modeling of non-inductive current drive and active control of instabilities. Considering these electron-cyclotron applied waves, it may be assumed that a time average over their characteristic high frequency scale results in the following low frequency kinetic equation ${ }^{30,31}$ :

$$
\frac{\partial f_{e}}{\partial t}+\mathbf{v} \cdot \frac{\partial f_{e}}{\partial \mathbf{x}}-\frac{e}{m_{e}}(\mathbf{E}+\mathbf{v} \times \mathbf{B}) \cdot \frac{\partial f_{e}}{\partial \mathbf{v}}=C_{e e}\left[f_{e}, f_{e}\right]+C_{e \iota}\left[f_{e}, f_{\iota}\right]+S^{R F}\left[f_{e}\right]
$$

The only difference between this and the kinetic equation the main body of this work was based on is the additional term $S^{R F}\left[f_{e}\right]$ that represents a quasilinear diffusion operator in velocity space and satisfies $\int d^{3} \mathbf{v} S^{R F}\left[f_{e}\right]=0$. Therefore, the analysis can proceed with minimal modifications if the amplitude of the applied waves is sufficiently small to make the RF source operator comparable to the collision operators, $S^{R F}\left[f_{e}\right] \simeq S^{R F}\left[f_{M e}\right] \sim \delta^{3}\left(v_{t h e} / L\right) f_{M e}$. Assuming this ordering, one can define the RF source moments

$$
\mathbf{F}_{e}^{R F}=m_{e} \int d^{3} \mathbf{v}\left(\mathbf{v}-\mathbf{u}_{e}\right) S^{R F}\left[f_{M e}\right]=O\left(\delta^{3} n m_{e} v_{t h e}^{2} / L\right)
$$

and

$$
G_{e}^{R F}=\frac{m_{e}}{2} \int d^{3} \mathbf{v}\left|\mathbf{v}-\mathbf{u}_{e}\right|^{2} S^{R F}\left[f_{M e}\right]=O\left(\delta^{3} n m_{e} v_{\text {the }}^{3} / L\right)
$$

Then, the fluid and drift-kinetic system is generalized by just adding the gyrophase average of the RF source operator, $\left\langle S^{R F}\left[f_{M e}\right]\right\rangle_{\alpha}$, to the right-hand-side of (26) and making the substitutions $\mathbf{F}_{e}^{\text {coll }} \rightarrow$ $\mathbf{F}_{e}^{\text {coll }}+\mathbf{F}_{e}^{R F}$ and $G_{e}^{\text {coll }} \rightarrow G_{e}^{\text {coll }}+G_{e}^{R F}$ in (10), (11) and (26). 


\section{References.}

${ }^{1}$ R.M. Kulsrud in Handbook of Plasma Physics, edited by M.N. Rosenbluth and R.Z. Sagdeev (NorthHolland, Amsterdam, 1983) Vol.1, p.115.

${ }^{2}$ R.D. Hazeltine and J.D. Meiss, Plasma Confinement (Addison Wesley, Redwood City, CA, 1992).

3 S. von Goeler, W. Stodiek and N. Sauthoff, Phys. Rev. Lett. 33, 1201 (1974).

${ }^{4}$ F. Porcelli, D. Boucher and M.N. Rosenbluth, Plasma Phys. Control. Fusion 38, 2163 (1996).

${ }^{5}$ Z. Chang, J.D. Callen, E.D. Frederickson, R.V. Budny, C.C. Hegna, K.M. McGuire, M.C. Zarnstorff and the TFTR group, Phys. Rev. Lett. 74, 4663 (1995).

${ }^{6}$ R. Carrera, R.D. Hazeltine and M. Kotschenreuther, Phys. Fluids 29, 899 (1986).

7 J.W. Connor, S.C. Cowley, R.J. Hastie and L.R. Pan, Plasma Phys. Control. Fusion 29, 919 (1987).

8 J.P. Wang and J.D. Callen, Phys. Fluids B 4, 1139 (1992).

9 Z.C. Chang and J.D. Callen, Phys. Fluids B 4, 1167 (1992).

${ }^{10}$ P.B. Snyder, G.W. Hammett and W. Dorland, Phys. Plasmas 4, 3974 (1997).

${ }^{11}$ P.J. Catto and A.N. Simakov, Phys. Plasmas 11, 90 (2004).

${ }^{12}$ E. D. Held, J.D. Callen, C.C. Hegna, C.R. Sovinec, T.A. Gianakon and S.E. Kruger, Phys. Plasmas 11, 2419 (2004).

${ }^{13}$ A. Dieter and R.D. Hazeltine, Phys. Plasmas 12, 052302 (2005).

${ }^{14}$ P. Goswami, T. Passot and P.L. Sulem, Phys. Plasmas 12, 102109 (2005).

${ }^{15}$ S.K. Wong and V.S. Chan, Phys. Plasmas 14, 112505 (2007).

${ }^{16}$ K.C. Shaing, Phys. Plasmas 14, 112509 (2007).

${ }^{17}$ E.A. Belli and J. Candy, Plasma Phys. Control. Fusion 50, 095010 (2008).

18 M.N. Rosenbluth, R.D. Hazeltine and F.L. Hinton, Phys. Fluids 15, 116 (1972).

19 F.L. Hinton and R.D. Hazeltine, Rev. Mod. Phys. 48, 239 (1976).

20 S.P. Hirshman and D.J. Sigmar, Nucl. Fusion 21, 1079 (1981).

21 J.J. Ramos, Phys. Plasmas 14, 052506 (2007).

22 L.D. Landau, Zh. Eksp. Teor. Fiz. 7, 203 (1937).

23 S. Chapman and T. Cowling, The Mathematical Theory of Non-Uniform Gases (Cambridge University Press, Cambridge, 1939). 
24 J.J. Ramos, Phys. Plasmas 15, 082106 (2008).

${ }^{25}$ M.N. Rosenbluth, W. MacDonald and D. Judd, Phys. Rev. 107, 1 (1957).

${ }^{26}$ P. Helander and D.J. Sigmar, Collisional Transport in Magnetized Plasmas (Cambridge University Press, Cambridge, 2002).

${ }^{27}$ O. Sauter, C. Angioni and Y.R. Lin-Liu, Phys. Plasmas 6, 2834 (1999).

${ }^{28}$ S.P. Hirshman, Phys. Fluids 21, 224 (1978).

${ }^{29}$ R.C. Morris, M.G. Haines and R.J. Hastie, Phys. Plasmas 3, 4513 (1996).

${ }^{30}$ C.C. Hegna and J.D. Callen, Phys. Plasmas 16, 112501 (2009).

${ }^{31}$ M. Brambilla, Kinetic Theory of Plasma Waves (Oxford University Press, Oxford, 1988). 